\title{
Comparison of capillary and venous blood for malaria detection using two PCR-based assays in febrile patients in Sierra Leone
}

\author{
Tomasz A. Leski ${ }^{1 *}$, Chris Rowe Taitt ${ }^{1}$, Umaru Bangura² ${ }^{2}$ Joseph Lahai ${ }^{2}$, Joseph M. Lamin² , Victoria Baio², \\ Mohamed S. Koroma², Abdulai G. Swaray ${ }^{3}$, Kathryn H. Jacobsen ${ }^{4}$, Olivia Jackson ${ }^{5}$, Brian W. Jones ${ }^{5}$, \\ Cynthia L. Phillips ${ }^{5}$, Rashid Ansumana ${ }^{2,6}$ and David A. Stenger ${ }^{1}$
}

\begin{abstract}
Background: Rapid and sensitive diagnostics are critical tools for clinical case management and public health control efforts. Both capillary and venous blood are currently used for malaria detection and while diagnostic technologies may not be equally sensitive with both materials, the published data on this subject are scarce and not conclusive.

Methods: Paired clinical samples of venous and capillary blood from 141 febrile individuals in Bo, Sierra Leone, were obtained between January and May 2019 and tested for the presence of Plasmodium parasites using two multiplexed PCR assays: the FilmArray-based Global Fever Panel (GFP) and the TaqMan-based Malaria Multiplex Sample Ready (MMSR) assay.

Results: No significant differences in Plasmodium parasite detection between capillary and venous blood for both assays were observed. The GFP assay was more sensitive than MMSR for all markers that could be compared (Plasmodium spp. and Plasmodium falciparum) in both venous and capillary blood.

Conclusions: No difference was found in malaria detection between venous and capillary blood using two different PCR-based detection assays. This data gives support for use of capillary blood, a material which can be obtained easier by less invasive methods, for PCR-based malaria diagnostics, independent of the platform.
\end{abstract}

Keywords: Malaria, Capillary blood, Venous blood, Multiplex polymerase chain reaction, Plasmodium, Plasmodium falciparum, Plasmodium vivax, Plasmodium ovale, Sierra Leone

\section{Background}

Malaria remains one of the most deadly infectious diseases in the world, disproportionately affecting lowerincome countries in Africa and South Asia. According to current World Health Organization (WHO) estimates, more than 200 million malaria cases and more than 400,000 deaths are expected to occur this year, with the

\footnotetext{
*Correspondence: tomasz.leski@nrl.navy.mil

${ }^{1}$ Center for Bio/Molecular Science \& Engineering, U.S. Naval Research Laboratory, Washington, DC, USA

Full list of author information is available at the end of the article
}

highest burden falling on young children in sub-Saharan Africa [1].

Sensitive and accurate diagnosis of malaria is the key to effective clinical case management and is an essential component of public health prevention and control strategies. Although diagnostic technologies using saliva and urine are being developed [2, 3], most current technologies continue to use whole blood collected via venipuncture or finger stick, and these materials are used interchangeably [4]. However, the chemical and cytological composition between blood from capillary and venous compartments differs [5-8], and these differences

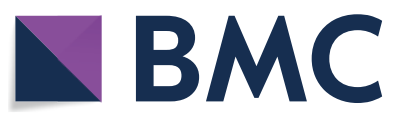

(c) The Author(s) 2021. This article is licensed under a Creative Commons Attribution 4.0 International License, which permits use, sharing, adaptation, distribution and reproduction in any medium or format, as long as you give appropriate credit to the original author(s) and the source, provide a link to the Creative Commons licence, and indicate if changes were made. The images or other third party material in this article are included in the article's Creative Commons licence, unless indicated otherwise in a credit line to the material. If material is not included in the article's Creative Commons licence and your intended use is not permitted by statutory regulation or exceeds the permitted use, you will need to obtain permission directly from the copyright holder. To view a copy of this licence, visit http://creativeco mmons.org/licenses/by/4.0/. The Creative Commons Public Domain Dedication waiver (http://creativecommons.org/publicdomain/ zero/1.0/) applies to the data made available in this article, unless otherwise stated in a credit line to the data. 
may potentially affect the sensitivity of malaria diagnostics. Sequestration of Plasmodium-infected erythrocytes in small capillaries of certain organs (e.g., brain, lungs) - a documented feature of malaria pathology [9] — may lead to unequal distribution of malaria parasites between capillary and venous compartments. That, in turn, may lead to different diagnostic results from these sample types.

Several previous studies assessed parasite density and the sensitivity of malaria detection in paired capillary and venous blood samples from asymptomatic carriers and symptomatic malaria patients in sub-Saharan Africa $[4,10-14]$. Some comparisons using standard microscopic techniques and PCR showed no difference between capillary and venous samples [10, 12, 13], but others showed slightly higher parasite numbers in capillary specimens $[4,11,14]$. Additionally, one PCR-based study documented a greater diversity of strains detected in venous blood samples [10]. Due to the lack of consensus regarding whether sensitivity is different for capillary and venous samples, this area of diagnostic research merits further investigation. This study compared the sensitivity of malaria detection in paired capillary and venous samples from symptomatic hospital patients in Bo, Sierra Leone, using two different PCR-based assays capable of detecting multiple Plasmodium species: the FilmArraybased Global Fever Panel (GFP) and the TaqMan-based Malaria Multiplex Sample Ready (MMSR) assay.

\section{Methods}

\section{Study population and sample collection}

The study population was recruited among persons seeking care at Mercy Hospital in Bo, Sierra Leone. All persons with clinically confirmed or self-reported fever with onset within the 10 days before the enrollment date were invited to participate in the study. Informed consent from patients (or, for children, consent from their parents) was obtained and documented prior to collection of clinical data and biological specimens. In total, 141 volunteers were enrolled between 28 January and 20 May 2019. Paired samples of venous and capillary blood were collected into EDTA-containing vacutainers or microtainers from each participant by venipuncture and finger prick, respectively. The study protocol was approved by the Institutional Review Boards at the US Naval Research Laboratory and George Mason University, and by the Sierra Leone Ministry of Health and Sanitation.

\section{PCR-based analysis}

Two PCR-based methods were used in this study: the FilmArray Global Fever Panel (GFP, BioFire Defense, Salt Lake City, UT, USA) and the Malaria Multiplex Sample Ready assay (MMSR, BioGX, Birmingham, AL, USA). The same volume $(200 \mu \mathrm{L})$ of blood specimen was used in both systems and from both specimen types. GFP is a fully automated, nested PCR assay capable of automated extraction of nucleic acids from a blood sample and rapid (one hour) detection of nineteen targets using the FilmArray platform, including P. falciparum, Plasmodium vivax/Plasmodium ovale, and Plasmodium spp. [15]. Briefly, within two hours of sample collection, $200 \mu \mathrm{L}$ of capillary or venous blood was mixed with $1 \mathrm{~mL}$ of GFP sample buffer. The entire diluted sample (total volume of $1.2 \mathrm{~mL}$ ) was loaded into GFP pouches, and analysed on the BioFire FilmArray 2.0 instrument according to the manufacturer's instructions. Detection and identification of the target is made automatically based on the melting temperature of the obtained amplicon. The validity of the run is determined by the system based on the amplification results of controls included in the assay pouch. The GFP has three different assays for Plasmodium: one species-level assay that detects $P$. falciparum, one specieslevel assay that detects both $P$. vivax and/or $P$. ovale, and one genus-level assay (Plasmodium spp.) that detects all Plasmodium species known to cause malaria in humans. The limits of detection (LOD) for GFP are 180, 150, and 240 genomic copies per $\mathrm{mL}$ blood for $P$. falciparum, $P$. vivax, and $P$. ovale, respectively (BioFire, unpublished data). The FilmArray system software calculates semiquantitative crossing point values (the fractional PCR cycle number when fluorescence of the sample exceeds the background fluorescence threshold). The manufacturer of the instrument refers to these values as " $\mathrm{Cp}$ values." The instrument's software does not provide these values to the user but they can be accessed by the manufacturer and were used in this study to help interpret the data. Since $\mathrm{Cp}$ values and the more commonly used term "Ct values" are different names for the same value, the term "Ct values" is used for both amplification systems for the purposes of the discussion in this paper. Due to the differences in PCR reaction chemistries between GFP and MMSR assays (two-step nested PCR vs. one-step TaqMan PCR, respectively), the Ct values obtained using GFP assay are generally lower MMSR Ct values for the same analyte concentrations and cannot be directly compared between these systems.

The MMSR assay is a room temperature-stabilized TaqMan-based real-time PCR assay [16, 17], designed to detect $P$. falciparum, $P$. vivax, Plasmodium spp., and RNaseP (sample extraction control) in a single assay. While this assay was not specifically designed to detect $P$. ovale and other less common malaria species, these parasites may be identified in samples testing positive for the genus specific marker (Plasmodium spp.) and negative for both species specific markers (P. falciparum and P. vivax) [18]. Within two hours of collection, DNA was extracted from $200 \mu \mathrm{L}$ capillary or venous blood using QIAamp 
DNA Mini Kit (Qiagen, Germantown, MD, USA); the final volume of extracted DNA was the same as the initial sample volume $-200 \mu \mathrm{L}$. Five microlitres of the extracted DNA was added to each MMSR tube, previously rehydrated with $5 \mu \mathrm{L}$ water. Tubes were then subjected to a thermal cycling program as follows: initial incubation at $95^{\circ} \mathrm{C}$ for 2 minutes; 45 cycles of denaturation at $95^{\circ} \mathrm{C}$ for 10 seconds and annealing/elongation at $59^{\circ} \mathrm{C}$ for $1 \mathrm{~min}$ ute. Fluorescence levels were measured at the end of each cycle. The samples were run using 8 -tube strips and each run contained a negative (no template) and a positive (P. falciparum DNA) control. The run results were considered valid when all controls gave expected results. A sample was considered positive for a particular target in the MMSR assay if a sigmoidal amplification curve with $\mathrm{Ct}$ value $<40$ was observed. The reported LODs for $P$. falciparum in the genus-specific assay (Plasmodium spp.) were 244-390 parasites per mL DNA solution, depending on whether the lyophilized or "wet" format was used [17]. The reported LOD in the P. falciparum-specific assay was similar in the lyophilized test (244 copies/mL), but was about 10-fold higher in the "wet" assay. The LOD for $P$. vivax was not reported for the lyophilized, "sample ready" format, but was previously determined for the "wet" assay as 127 parasites/mL [16].

Data from capillary or venous samples with negative RNaseP results (MMSR assay) or invalid FilmArray results were not included in the comparison. However, if the matched partner of the invalid sample showed valid results, data from the matched partner were included in statistical analyses of the population as a whole.

Sample populations returning valid results were compared using McNemar's chi-square analysis of (self-) paired samples, corrected for continuity. Ct values for positive (matched) samples were compared using paired t-tests and for unmatched populations using unpaired $\mathrm{t}$-tests, assuming unequal variances.

\section{Results}

The median age of the 141 participants that provided blood samples was 27 years (range: 5 to 78 years), and $60 \%$ were female. Depending on the assay and material tested, at least one malaria marker was detected in $36-61 \%$ of the tested samples (Table 1). These results were in line with previous reports of malaria positivity among febrile individuals in this location as detected by PCR-based methods $[18,19]$.

The study was designed to return four test results for each participant (two platforms, both venous and capillary samples tested on each). However approximately $63 \%$ of the participants had one or more missing data points primarily due to insufficient sample volumes to perform both tests on both platforms or invalid runs
Table 1 Malaria marker prevalence as detected by GFP and MMSR assays for paired venous/capillary samples

\begin{tabular}{lcc}
\hline & \multicolumn{2}{c}{ Number (\%) of positive samples } \\
\cline { 2 - 3 } & $\begin{array}{l}\text { GFP } \\
(\mathbf{n}=\mathbf{6 6})\end{array}$ & $\begin{array}{l}\text { MMSR } \\
(\mathbf{n}=\mathbf{1 1 0})\end{array}$ \\
\hline Capillary & & $29(26 \%)$ \\
Plasmodium spp. & $37(56 \%)$ & $37(34 \%)$ \\
P. falciparum & $32(48 \%)$ & 0 \\
P. vivax/P. ovale & $4(6 \%)$ & $40(36 \%)$ \\
Any malaria marker & $38(58 \%)$ & $35(32 \%)$ \\
Venous & & $37(34 \%)$ \\
Plasmodium spp. & $40(61 \%)$ & 0 \\
P. falciparum & $30(45 \%)$ & $40(36 \%)$ \\
P. vivax/P. ovale & $4(6 \%)$ & \\
Any malaria marker & $40(61 \%)$ & \\
\hline a GFP assay detects both P. vivax and P. ovale while MMSR assay detects only P.
\end{tabular}
vivax

(27 samples). The most common causes of the invalid runs were failures of the extraction control reactions for MMSR (3 samples) and hardware issues with one of the two FilmArray instruments used in case of GFP assay (18 samples). Consequently, valid results were returned for 110 paired capillary/venous blood samples using MMSR and for 66 paired samples using GFP. For cross-platform comparisons, venous samples from 136 individuals and capillary samples from 54 individuals were successfully tested on both platforms. The demographic and clinical characteristics of included and excluded volunteers were similar.

No significant age-specific differences were observed for any marker on either platform (Additional file 1: Table S1). Although no gender-specific differences were noted on the GFP platform, a significantly higher proportion of male participants were positive for Plasmodium $\mathrm{spp}$. in the MMSR assay $(\mathrm{p}=0.011)$; this result may simply represent an artifact of the large relative difference between male and female sample sets within a small total population $(\mathrm{n}=136)$.

\section{Comparison of detection platforms}

A total of 54 matched capillary and 136 venous blood samples returned valid results for both GFP and MMSR. The results for both detection platforms were compared in Table 2 for capillary and Table 3 for venous samples. GFP assays detected a significantly higher proportion of positive samples across all detected markers for both capillary and venous blood. The difference was particularly evident with the genus-level marker (Plasmodium spp.). The GFP platform detected Plasmodium spp. in over half of the samples (both sample types), whereas the same 
Table 2 Comparison of malaria detection by GFP and MMSR assays - capillary blood $(n=54)$; GFP is more sensitive than MMSR

\begin{tabular}{|c|c|c|c|c|c|}
\hline \multicolumn{5}{|l|}{ Marker } & \multirow{2}{*}{$\begin{array}{l}\text { p-value for McNemar's } \mathbf{X}^{2} \text { test } \\
<0.001\left(x^{2}=13.474\right)\end{array}$} \\
\hline Plasmodium spp. & & MMSR & & & \\
\hline \multirow[t]{4}{*}{ GFP } & & Positive & Negative & Total & \\
\hline & Positive & 10 & 18 & 28 & \\
\hline & Negative & 1 & 25 & 26 & \\
\hline & Total & 11 & 43 & 54 & \\
\hline P. falciparum & & MMSR & & & $0.043\left(x^{2}=4.083\right)$ \\
\hline \multirow[t]{4}{*}{ GFP } & & Positive & Negative & Total & \\
\hline & Positive & 13 & 10 & 23 & \\
\hline & Negative & 2 & 29 & 31 & \\
\hline & Total & 15 & 39 & 54 & \\
\hline Any Plasmodium marker & & MMSR & & & $0.006\left(x^{2}=7.563\right)$ \\
\hline \multirow[t]{4}{*}{ GFP } & & Positive & Negative & Total & \\
\hline & Positive & 14 & 14 & 28 & \\
\hline & Negative & 2 & 24 & 26 & \\
\hline & Total & 16 & 38 & 54 & \\
\hline
\end{tabular}

Table 3 Comparison of malaria detection by GFP and MMSR assays-venous blood $(n=136)$; GFP is more sensitive than MMSR

\begin{tabular}{|c|c|c|c|c|c|}
\hline \multicolumn{5}{|l|}{ Marker } & \multirow{2}{*}{$\begin{array}{l}p \text {-value for McNemar's } \boldsymbol{x}^{2} \text { test } \\
<0.001\left(X^{2}=25.037\right)\end{array}$} \\
\hline Plasmodium spp. & & MMSR & & & \\
\hline \multirow[t]{4}{*}{ GFP } & & Positive & Negative & Total & \\
\hline & Positive & 44 & 27 & 71 & \\
\hline & Negative & 0 & 65 & 65 & \\
\hline & Total & 44 & 92 & 136 & \\
\hline P. falciparum & & MMSR & & & $0.022\left(x^{2}=5.263\right)$ \\
\hline \multirow[t]{4}{*}{ GFP } & & Positive & Negative & Total & \\
\hline & Positive & 43 & 15 & 58 & \\
\hline & Negative & 4 & 74 & 78 & \\
\hline & Total & 47 & 89 & 136 & \\
\hline Any malaria marker & & MMSR & & & $<0.001\left(x^{2}=17.391\right)$ \\
\hline \multirow[t]{4}{*}{ GFP } & & Positive & Negative & Total & \\
\hline & Positive & 49 & 22 & 71 & \\
\hline & Negative & 1 & 64 & 65 & \\
\hline & Total & 50 & 86 & 136 & \\
\hline
\end{tabular}

marker was detected in $22 \%$ fewer samples analysed by MMSR. Fewer samples were positive for P. falciparum marker than Plasmodium spp. marker on the GFP, but cross-platform differences were still significant ( $P$. falciparum was detected in 23/54 capillary samples by GFP compared to only $15 / 54$ by MMSR, and $58 / 136$ venous samples by GFP compared to only $47 / 136$ by MMSR). In the case of MMSR assays, a smaller number of samples tested positive for Plasmodium spp. marker than P. falciparum marker for both sample types; this likely reflects previously reported differences in LOD values for these markers $[16,17]$. Power analyses indicated that the sample sizes were sufficient to determine platform differences $(80 \%$ power, $\alpha=0.05)$ in detection of Plasmodium spp. using any marker; however, sample sizes 1.3- to 1.6times greater would be required for platform differences in detection of $P$. falciparum and any malaria marker with the same power and significance.

The GFP assay detected $P$. vivax/P. ovale in six out of 136 matched venous samples $(4 \%)$ and in four out of 54 matched capillary samples $(7 \%)$. The four capillary samples positive for $P$. vivax/P. ovale were simultaneously 
positive for this marker in venous samples. However, none of these samples were deemed positive for $P$. vivax in the MMSR assay, suggesting that the $P$. ovale may have been responsible for the $P$. vivax/P. ovale-positives in the GFP assay.

P. ovale (and Plasmodium malariae) may be detected, in the MMSR assay, when a sample is Plasmodium spp.-positive and negative for the species-specific markers [18]; only one of the samples positive by GFP for $P$. vivax/P. ovale demonstrated this behavior in the MMSR assay. Presumptively, samples harbouring $P$. malariae and other less-common species can be identified similarly by the MMSR assay (negative for $P$. vivax/P. falciparum and positive for Plasmodium spp.). This does not have to be true, however, for GFP because the Plasmodium spp. assay is slightly more sensitive than the GFP species-level assays (BioFire, unpublished data).

An additional nine venous and four capillary samples were identified that were Plasmodium spp.-positive but negative in the species-specific assays in GFP assays; all but two were negative for all markers in the corresponding MMSR assays. Interestingly, only three had identical results in both venous and capillary samples. All eleven of these samples that were only positive for Plasmodium spp. had late $\mathrm{Ct}$ values, suggesting analyte levels near LOD where it is expected that the Plasmodium spp. assay will slightly outperform the species-level assays on the GFP.

\section{Comparison of venous and capillary samples}

Results from matched sets of capillary and venous samples using GFP (Table 4) showed no significant differences in the proportion of samples testing positive for any of the malaria markers between venous and capillary samples. Fewer than $10 \%$ of the 66 matched samples on GFP gave discordant results where a marker was positive in one sample type and negative in the other. Comparisons of $\mathrm{Ct}$ values between venous and capillary samples (Fig. 1) show a high degree of correlation when tested using GFP. There was no significant difference in $\mathrm{Ct}$ values between matched venous and capillary samples for any marker $(\mathrm{p}>0.13)$.

Similarly, no significant differences between positive results were observed for any marker when using MMSR ( $p>0.24, n=110$; Table 5). There was lower concordance between the two sample types in MMSR assays than in the GFP assays $-82 \%$ for genus specific marker (Plasmodium spp.), $84 \%$ for P. falciparum and $84 \%$ for any malaria marker versus $>92 \%$ for GFP. A lower correlation was observed when comparing $\mathrm{Ct}$ values for matched samples in MMSR assays (Fig. 2), although no significant differences in $\mathrm{Ct}$ values were observed, independent of the assay $(p>0.6)$. However,
Table 4 Comparison of malaria detection in capillary and venous blood-GFP assay $(n=66)$

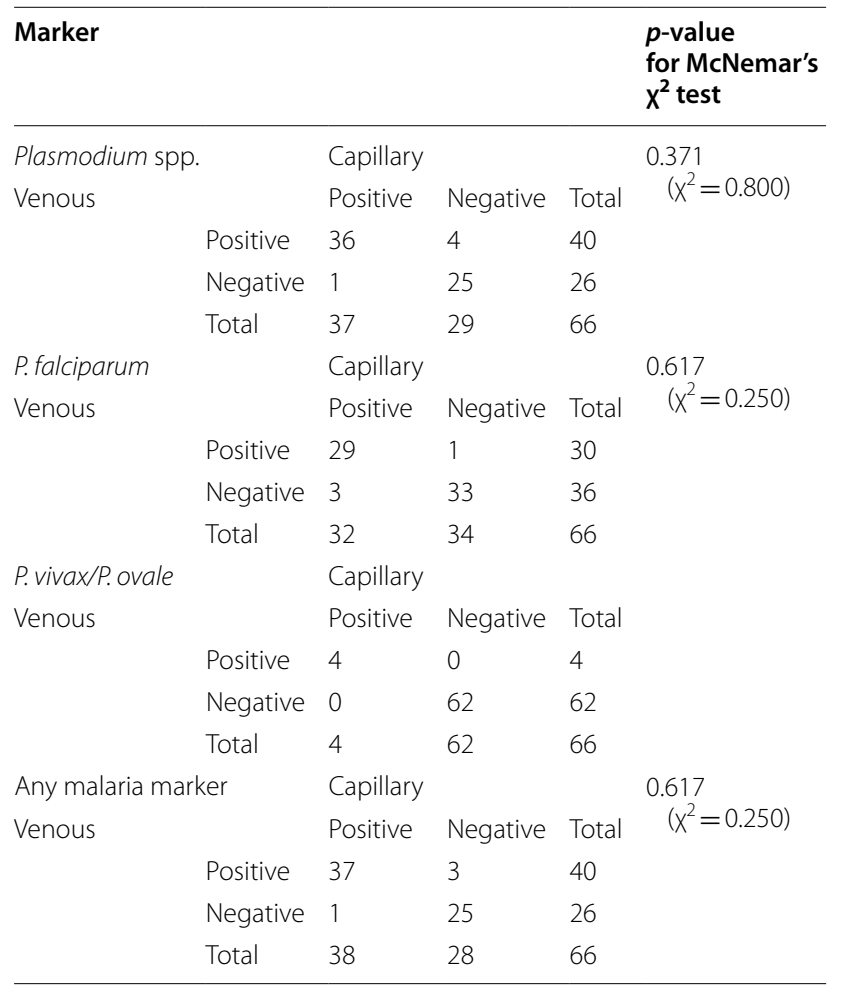

when comparing concordant populations (both sample types positive) and discordant populations (one sample type positive, one sample type negative), $\mathrm{Ct}$ values from the discordant populations were indeed higher ( $p<0.015$, see Fig. 1), supporting the hypothesis that samples with discordant results had lower target concentrations.

Percentages of discordant samples ranged from 1.5 to $6.1 \%$ in the GFP assays and $6.4-11.8 \%$ in the MMSR assays. Given similar prevalence of venous-positive/ capillary-negative and venous-negative/capillary-positive pairs, power analyses indicated that we would need to test at least four times as many samples to show differences between the two sample types with $\alpha=0.05$ and $80 \%$ power for all three GFP assays and MMSRbased Plasmodium spp. detection; identical rates of detection from both sample types in MMSR-based detection of $P$. falciparum and any malaria marker indicate that a much larger sample size $(n>>1000)$ would be needed to detect differences between sample types.

\section{Discussion}

The goal of this study was to compare the sensitivity of DNA-based malaria diagnostic assays using capillary and venous blood. In contrast to most of the previous studies 

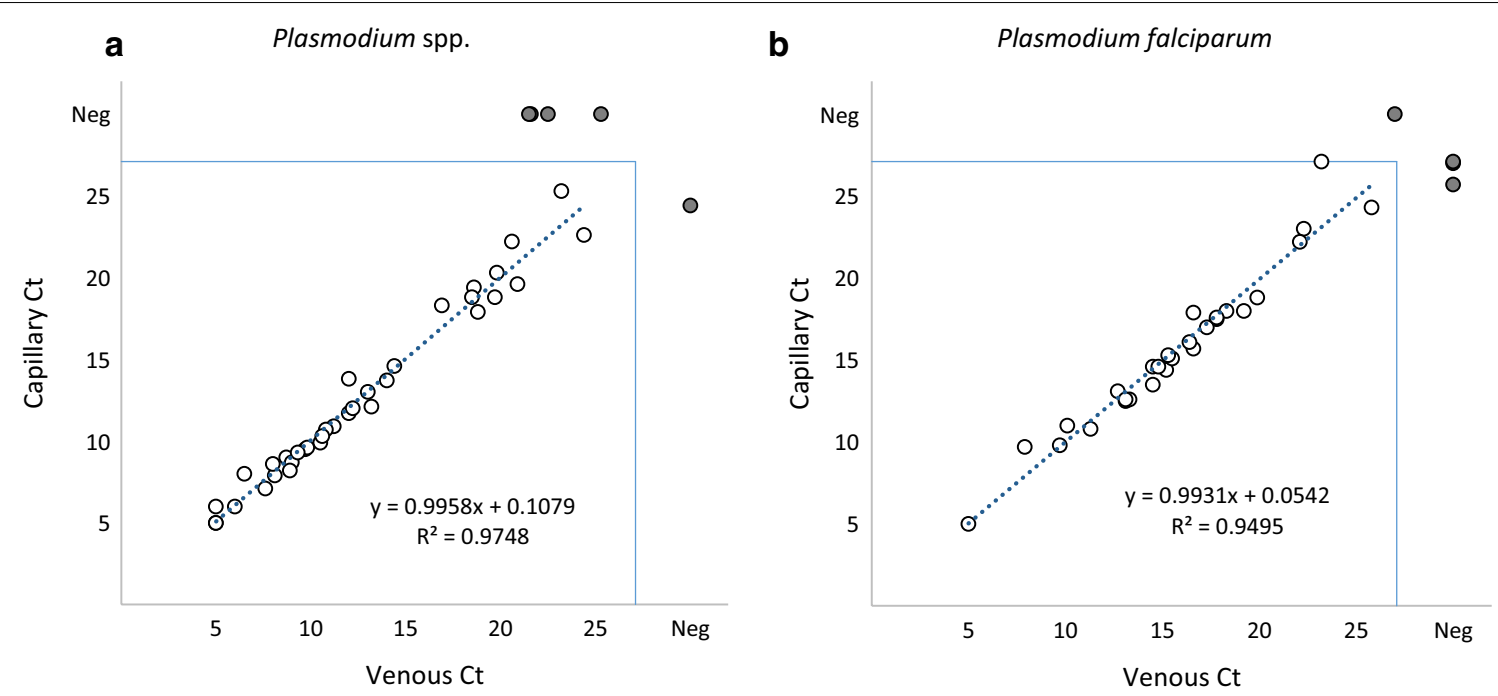

Fig. 1 Comparison of Ct values obtained with venous (abscissa) and capillary (ordinate) samples positive for malaria markers after analysis using GFP. Panels A and B show Ct values for samples positive for Plasmodium spp. (a) and P. falciparum (b). Gray circles at the edges of each graph represent samples that were positive in venous but not matched capillary samples (above the main plot) or in capillary but not matched venous samples (to the right of each plot)

Table 5 Comparison of malaria detection in capillary and venous blood - MMSR assay $(n=110)$

\begin{tabular}{|c|c|c|c|c|c|}
\hline \multicolumn{5}{|l|}{ Marker } & \multirow{2}{*}{$\begin{array}{l}\boldsymbol{p} \text {-value for McNemar's } \boldsymbol{X}^{2} \text { test } \\
0.264\left(X^{2}=1.250\right)\end{array}$} \\
\hline Plasmodium spp. & & Capillary & & & \\
\hline \multirow[t]{4}{*}{ Venous } & & Positive & Negative & Total & \\
\hline & Positive & 22 & 13 & 35 & \\
\hline & Negative & 7 & 68 & 75 & \\
\hline & Total & 29 & 81 & 110 & \\
\hline P. falciparum & & Capillary & & & $0.814\left(x^{2}=0.0556\right)$ \\
\hline \multirow[t]{4}{*}{ Venous } & & Positive & Negative & Total & \\
\hline & Positive & 28 & 9 & 37 & \\
\hline & Negative & 9 & 64 & 73 & \\
\hline & Total & 37 & 73 & 110 & \\
\hline P. vivax & & Capillary & & & \\
\hline \multirow[t]{4}{*}{ Venous } & & Positive & Negative & Total & \\
\hline & Positive & 0 & 0 & 0 & \\
\hline & Negative & 0 & 110 & 110 & \\
\hline & Total & 0 & 110 & 110 & \\
\hline Any malaria marker & & Capillary & & & $0.814\left(x^{2}=0.0556\right)$ \\
\hline \multirow[t]{4}{*}{ Venous } & & Positive & Negative & Total & \\
\hline & Positive & 31 & 9 & 40 & \\
\hline & Negative & 9 & 61 & 70 & \\
\hline & Total & 40 & 70 & 110 & \\
\hline
\end{tabular}

comparing these two sample types [4, 10-13], the sensitivity of two PCR assays capable of detecting multiple species of Plasmodium was assessed instead of determining the parasite density by microscopy. While PCR and other nucleic acid based assays are not currently used for routine malaria diagnostics in most countries, these techniques are increasingly used for epidemiological studies of malaria and there is a growing recognition of their important role in detecting subclinical malaria infections especially when coupled with strategic 

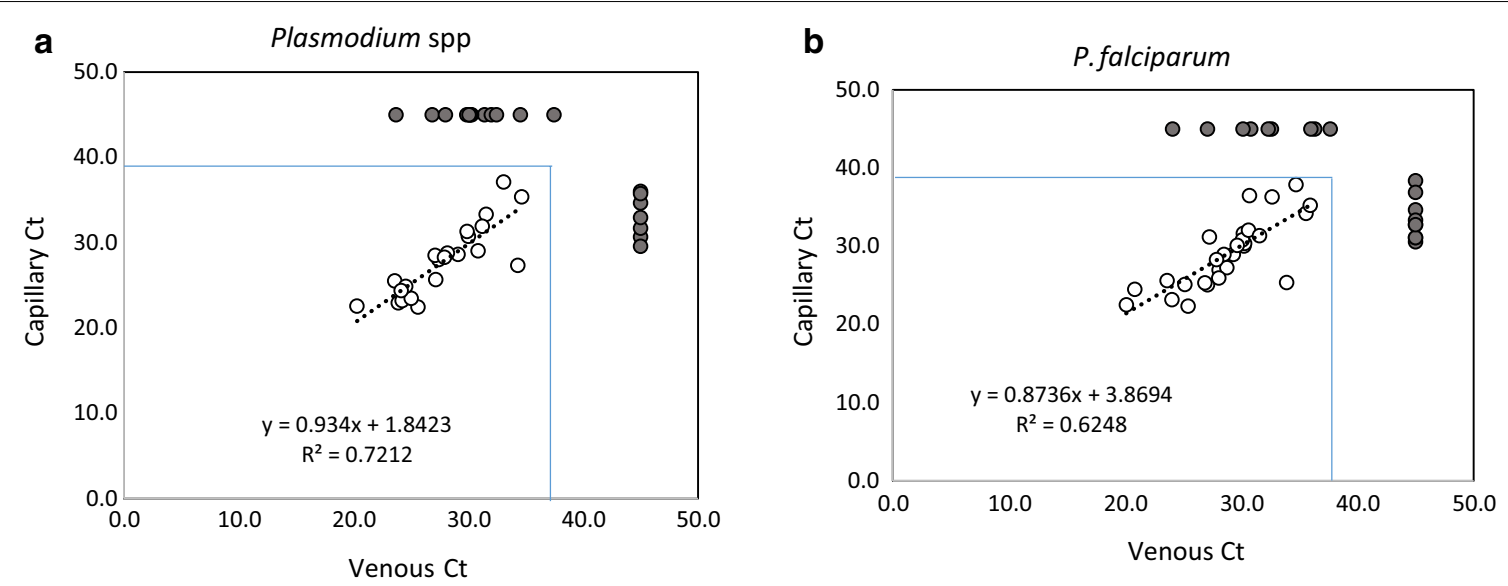

Fig. 2 Comparison of Ct values obtained with venous (abscissa) and capillary (ordinate) samples positive for malaria markers after analysis using MMSR. Panels A and B show Ct values for samples positive for Plasmodium spp. (a) and P. falciparum (b). Gray circles at the edges of each graph represent samples that were positive in venous but not matched capillary samples (above the main plot) or in capillary but not matched venous samples (to the right of each plot)

malaria elimination and eradication efforts. The analysis described here documented no significant differences in malaria detection between capillary and venous blood using two different PCR based assays.

These results are in line with several previous studies that also found no differences in parasite density or malaria detection between capillary and venous samples $[10,12,13]$. Comparisons of $\mathrm{Ct}$ values obtained using GFP and MMSR further support the hypothesis that there are no significant differences in average parasite densities between these two sample types.

The higher malaria detection rate observed for GFP compared to MMSR for all detected markers reflects fundamental and comprehensive differences between the two systems: different sample processing (nucleic acid extraction), different PCR chemistry, nested PCR (GFP) versus single-step PCR (MMSR), and different gene targets. Although the previously determined LODs for the two platforms are within the same range $(\sim 150-400$ parasites or target copies per millilitre), a five-fold larger MMSR sample volume was used in the current study than in the MMSR LOD study ( $5 \mu \mathrm{L}$ versus $1 \mu \mathrm{L}$ [17]); it is possible that the increased sample volume, combined with a different method for DNA extraction, interfered with the MMSR assays performed here. The differences in sensitivity can be also a consequence of using different diagnostic targets; the effect is especially pronounced in case of the genus-specific targets (Plasmodium spp.). The samples with discordant results between sample types had significantly higher $\mathrm{Ct}$ values on both platforms; these results suggest that discordant results may arise when testing samples with parasite concentrations close to the LOD.

It was not possible to unambiguously assess the sensitivity differences in detecting $P$. vivax (MMSR) and $P$. vivax/P. ovale (GFP) due to the different specificities of the two platforms and the low prevalence of $P$. vivax within West Africa [1, 20, 21]. Importantly, two of the samples that were only positive for Plasmodium spp. by GFP in venous blood had relatively early $\mathrm{Ct}$ values that, if the infection were $P$. falciparum, $P$. ovale, or $P$. vivax, should also have been detected by one of the specieslevel assays; it is therefore likely that these samples contained $P$. malariae. The presence of small but significant numbers of samples positive for $P$. vivax/P. ovale in the GFP assay or Plasmodium spp. only in both assays could be due to co-circulation of $P$. ovale and P. malariae in the tested population as previously reported [18].

Three studies have previously reported higher parasite densities or improved malaria detection sensitivities in capillary blood $[4,11,12]$. The hypothesis that capillary blood may contain higher concentrations of malaria parasites is based on the well-documented feature of malaria pathology-increased adherence of Plasmodium-infected erythrocytes to vascular endothelial cells-which leads to their sequestration in certain organs [9]. However, while it is known that malaria-infected erythrocytes can sequester in specific organs (e.g., brain, lungs), it is not clear if significant sequestration occurs in capillaries used for diagnostic sample collection. The results obtained in this study do not provide support to the hypothesis that Plasmodium markers are present in higher concentrations in capillary blood, but rather, adds to the body of 
literature showing no documented difference between sample types.

A notable difference between the current and previous studies is the population age distribution. Whereas most other studies emphasized testing of children or young teens $[4,10,11,13,14]$, our study population was significantly older, with a median of 27 years and $25 \%$ of the tested individuals between 21 and 25 years of age. It is possible that age-related physiological differences may affect the parasite's life cycle, its clinical presentation, and potentially even its prevalence within various compartments [22-24]. An age-controlled study with a greater number of participants and longer collection period would be needed to explore these variables.

\section{Conclusions}

In summary, no difference in Plasmodium parasite detection sensitivity between capillary and venous blood was found. While accepting the limitations of the current study (small sampled population with a different age distribution from previous studies), the results presented here provide additional supporting evidence that PCR-based methods can produce equally satisfactory results using capillary and venous blood, in spite of the higher diagnostic sensitivity of capillary blood suggested by some recent studies. The implication of this finding is that capillary blood-typically less cumbersome and invasive to obtain - can be used without a loss of the assay sensitivity. Additional, larger-scale studies are needed to support these conclusions.

\section{Supplementary Information}

The online version contains supplementary material available at https://doi. org/10.1186/s12936-021-03644-y.

Additional file 1: Table S1. Samples positive for various malaria markers by age and gender

\section{Abbreviations}

Cp: Crossing point.; Ct: Crossing threshold.; GFP: Global Fever Panel.; MMSR: Multiplex Malaria Sample Ready assay;; LOD: Limit of detection.; PCR: Polymerase chain reaction.; WHO: World Health Organization..

\section{Acknowledgements}

Not applicable.

\section{Authors' contributions}

TAL, CRT, RA, DAS conceived the study and designed the experimental protocols; RA, UB, JL, JML, VB, RA performed the laboratory testing; AGS processed the experimental data and developed the database; TAL, CRT, KHJ developed human subjects protocol; OJ, BWJ, CLP analysed the GFP assay data; CRT performed the statistical analyses; TAL and CRT wrote the first version of the manuscript; UB, OJ, BWJ, CLP, RA, KHJ, DAS made edits to the manuscript. All authors read and approved the final manuscript.

\section{Funding}

This work was supported by the Joint Science and Technology Office (JSTO), Defense Threat Reduction Agency (DTRA). This manuscript is approved for public release: distribution is unlimited. The funding body played no role in the design of the study and collection, analysis, and interpretation of data and in writing the manuscript.

\section{Availability of data and material}

The datasets used and/or analyzed during the current study are available from the corresponding author on reasonable request.

\section{Ethics approval and consent to participate}

The research protocol used in this study was approved by the Sierra Leone Ethics and Scientific Review Committee and the institutional review boards of the US Naval Research Laboratory and George Mason University.

\section{Consent for publication}

Not applicable.

\section{Competing interests}

Brian Jones, Olivia Jackson and Cynthia Phillips are employees of BioFire Defense, the manufacturer of the instrument (FilmArray) and assay (Global Fever Panel) used in this study.

\section{Author details}

${ }^{1}$ Center for Bio/Molecular Science \& Engineering, U.S. Naval Research Laboratory, Washington, DC, USA. ${ }^{2}$ Mercy Hospital Research Laboratory, Bo, Sierra Leone. ${ }^{3}$ College of Science, George Mason University, Fairfax, Virginia, USA.

${ }^{4}$ Department of Global \& Community Health, George Mason University, Fairfax, Virginia, USA. ${ }^{5}$ BioFire Defense, Salt Lake City, Utah, USA. ${ }^{6}$ Njala University, Bo, Sierra Leone.

Received: 29 January 2020 Revised: 8 February 2021 Accepted: 10 February 2021

Published online: 06 March 2021

\section{References}

1. WHO. World malaria report 2019. Geneva: World Health Organization, 2019.

2. Putaporntip C, Buppan $P$, Jongwutiwes S. Improved performance with saliva and urine as alternative DNA sources for malaria diagnosis by mitochondrial DNA-based PCR assays. Clin Microbiol Infect. 2011;17:1484-91.

3. Tao D, McGill B, Hamerly T, Kobayashi T, Khare P, Dziedzic A, et al. A salivabased rapid test to quantify the infectious subclinical malaria parasite reservoir. Sci Transl Med. 2019;11:eaan4479.

4. Mischlinger J, Pitzinger P, Veletzky L, Groger M, Zoleko-Manego R, Adegnika AA, et al. Use of capillary blood samples leads to higher parasitemia estimates and higher diagnostic sensitivity of microscopic and molecular diagnostics of malaria than venous blood samples. J Infect Dis. 2018;218:1296-305.

5. Daae LN, Halvorsen S, Mathisen PM, Mironska K. A comparison between haematological parameters in 'capillary' and venous blood from healthy adults. Scand J Clin Lab Invest. 1988;48:723-6.

6. Daae LN, Hallerud M, Halvorsen S. A comparison between haematological parameters in 'capillary' and venous blood samples from hospitalized children aged 3 months to 14 years. Scand J Clin Lab Invest. 1991;51:651-4.

7. Yang ZW, Yang SH, Chen L, Qu J, Zhu J, Tang Z. Comparison of blood counts in venous, fingertip and arterial blood and their measurement variation. Clin Lab Haematol. 2001;23:155-9.

8. Kupke IR, Kather B, Zeugner S. On the composition of capillary and venous blood serum. Clin Chim Acta. 1981;112:177-85.

9. Ockenhouse CF, Ho M, Tandon NN, Van Seventer GA, Shaw S, White NJ, et al. Molecular basis of sequestration in severe and uncomplicated Plasmodium falciparum malaria: differential adhesion of infected erythrocytes to CD36 and ICAM-1. J Infect Dis. 1991;164:163-9. 
10. Lehane A, Were M, Wade M, Hamadu M, Cahill M, Kiconco S, et al. Comparison on simultaneous caillary and venous parasite density and genotyping results from children and adults with uncomplicated malaria: a prospective observational study in Uganda. BMC Infect Dis. 2019;19:559.

11. Ouédraogo JB, Guigemdé TR, Gbary AR. Etude comparative de la densite parasitaire de Plasmodium falciparum dans le sang capillaire et dans le sang veineux chez des porteurs asymptomatiques (région de BoboDioulasso, Burkina Faso). Médecine d'Afrique Noire. 1991;38:601-5.

12. Njunda A, Assob N, Nsagha S, Kamga F, Mokenyu M, Kwenti E. Comparison of capillary and venous blood using blood film microscopy in the detection of malaria parasites: a hospital based study. Sci J Microbiol. 2013;2:89-94.

13. Sandeu MM, Bayibeki AN, Tchioffo MT, Abate L, Gimonneau G, AwonoAmbene $\mathrm{PH}$, et al. Do the venous blood samples replicate malaria parasite densities found in capillary blood? A field study performed in naturally-infected asymptomatic children in Cameroon. Malar J. 2017; 16:345.

14. Kast K, Berens-Riha N, Zeynudin A, Abduselam N, Eshetu T, Loscher T, et al. Evaluation of Plasmodium falciparum gametocyte detection in different patient material. Malar J. 2013;12:438.

15. Helm JR, Toxopeus C, Batty N, Border L, Davidson O, Kelley A, et al. Analytical performance of the FilmArray ${ }^{\circledR}$ global fever panel. Presented at the American Society of Tropical Medicine and Hygiene Annual Meeting 2018: Poster 1445. https://www.biofiredefense.com/wp-content/uploa ds/2018/11/Analytical-Performance-of-the-FilmArray-Global-Fever-Panel -ASTMH-Poster-1.pdf.

16. Kamau E, Alemayehu S, Feghali KC, Saunders D, Ockenhouse CF. Multiplex qPCR for detection and absolute quantification of malaria. PLoS One. 2013;8:e71539.
17. Kamau E, Alemayehu S, Feghali KC, Juma DW, Blackstone GM, Marion WR, et al. Sample-ready multiplex qPCR assay for detection of malaria. Malar J. 2014;13:158

18. Leski TA, Taitt CR, Swaray AG, Bangura U, Reynolds N, Holtz A, et al. Use of real-time multiplex $P C R$, malaria rapid diagnostic test and microscopy to investigate the prevalence of Plasmodium species among febrile hospital patients in Sierra Leone. Malar J. 2020;19:84.

19. Mulvaney SP, Fitzgerald LA, Hamdan LJ, Ringeisen BR, Petersen ER, Compton JR, et al. Rapid design and fielding of four diagnostic technologies in Sierra Leone, Thailand, Peru, and Australia: successes and challenges faced introducing these biosensors. Sensing Bio-Sensing Res. 2018;20:22-33.

20. Poirier P, Doderer-Lang C, Atchade PS, Lemoine JP, de I'Isle MC, AbouBacar A, et al. The hide and seek of Plasmodium vivax in West Africa: report from a large-scale study in Beninese asymptomatic subjects. Malar J. 2016;15:570.

21. Oboh MA, Badiane AS, Ntadom G, Ndiaye YD, Diongue K, Diallo MA, et al. Molecular identification of Plasmodium species responsible for malaria reveals Plasmodium vivax isolates in Duffy negative individuals from southwestern Nigeria. Malar J. 2018;17:439.

22. Hawkes M, Elphinstone RE, Conroy AL, Kain KC. Contrasting pediatric and adult cerebral malaria: the role of the endothelial barrier. Virulence. 2013;4:543-55.

23. Ashley EA, Pyae Phyo A, Woodrow CJ. Malaria. Lancet. 2018;391:1608-21.

24. Clark IA, Alleva LM, Mills AC, Cowden WB. Pathogenesis of malaria and clinically similar conditions. Clin Microbiol Rev. 2004;17:509-39.

\section{Publisher's note}

Springer Nature remains neutral with regard to jurisdictional claims in published maps and institutional affiliations.
Ready to submit your research? Choose BMC and benefit from:

- fast, convenient online submission

- thorough peer review by experienced researchers in your field

- rapid publication on acceptance

- support for research data, including large and complex data types

- gold Open Access which fosters wider collaboration and increased citations

- maximum visibility for your research: over 100M website views per year

At BMC, research is always in progress.

Learn more biomedcentral.com/submissions 